International Journal of Pure and Applied Mathematics

Volume $95 \quad$ No. 3 2014, 357-366

ISSN: 1311-8080 (printed version); ISSN: 1314-3395 (on-line version)

url: http://www.ijpam.eu

doi: http://dx.doi.org/10.12732/ijpam.v95i3.4

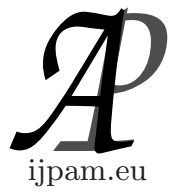

\title{
EXACT SOLUTION OF THE CLASSICAL SU(2) YANG-MILLS FIELD EQUATIONS
}

\author{
A.R. Shehata ${ }^{1}$, J.F. Alzaidy ${ }^{2}$ \\ ${ }^{1}$ Mathematics Department \\ Faculty of Science \\ Minia University \\ El-Minia, EGYPT \\ ${ }^{2}$ Mathematics Department \\ Faculty of Science \\ Taif University \\ KINGDOM OF SAUDI ARABIA
}

\begin{abstract}
In this paper we find a new representation for self-duality equations. In addition exact solution class of the classical $S U(2)$ Yang-Mills field equations in four-dimensional Euclidean space and two exact solution classes for $S U(2)$ Yang- Mills equations when is $\rho$ a complex analytic function are also obtained.
\end{abstract}

AMS Subject Classification: 35, 53C, 58J, 58Z05

Key Words: self-dual $S U(2)$, Yang-Mills fields, Gauge theory

\section{Introduction}

The self-dual Yang-Mills equations (a system of equations for Lie algebra valued functions of $C^{4}$ ) play a central role in the field of integrable systems and also play a fundamental role in several other areas of mathematics and physics, see [1]-[4]. In addition the self-dual Yang-Mills equations are of great importance in

Received: March 23, 2014

(C) 2014 Academic Publications, Ltd.

${ }^{\S}$ Correspondence author url: www.acadpubl.eu 
their own right and have found a remarkable number of applications in physics and mathematics as well. These equations arise in the context of gauge theory (see [5]), in classical general relativity (see [6], [7]), and can be used as a powerful tool in the analysis of 4-manifolds, see [8]. The Yang-Mills equations are a set of coupled, second-order partial differential equations in four dimensions for the Lie algebra-valued gauge potential functions $A_{\mu}$, and are extremely difficult to solve in general. The self-dual Yang-Mills equations describe a connection for a bundle over the Grassmannian of two-dimensional subspaces of the twistor space, see [9], [10].

In this paper we found a new representation for self-duality equations. In addition exact solution class of the classical $S U(2)$ Yang-Mills field equations in four-dimensional Euclidean space and two exact solution classes for $S U(2)$ Yang-Mills equations when $\rho$ is a complex analytic function are also obtained. This paper is organized as follows: This introduction followed by the new representation of the self-duality equations in Section 2. In Section 3 we found an exact solution class of the classical $S U(2)$ Yang-Mills field equations. Moreover two exact solution classes for self-dual $S U(2)$ gauge fields on Euclidean space when $\rho$ is a complex analytic function are given in Section 4. Finally, we give some conclusions in Section 5 .

\section{New Representation of the Self-Duality Equations}

The essential idea of Yang and Mills (1954) [11] is to consider an analytic continua-tion of the gauge potential $A_{\mu}$ into complex space where $x_{1}, x_{2}, x_{3}$ and $x_{4}$ are complex. The self-duality equations $F_{\mu \nu}={ }^{*} F_{\mu \nu}$ are then valid also in complex space, in a region containing real space where the $x$ 's are real. Now consider four new complex variables $y, \bar{y}, z$ and $\bar{z}$ defined by

$$
\begin{array}{ll}
\sqrt{2} y=x_{1}+i x_{2}, & \sqrt{2} \bar{y}=x_{1}-i x_{2}, \\
\sqrt{2} z=x_{3}-i x_{4}, & \sqrt{2} \bar{z}=x_{3}+i x_{4},
\end{array}
$$

it is simple to check that the self-duality equations $F_{\mu \nu}={ }^{*} F_{\mu \nu}$ reduces to

$$
F_{y z}=0, \quad F_{\bar{y} \bar{z}}=0, \quad F_{y \bar{y}}+F_{z \bar{z}}=0 .
$$

Equations (2) can be immediately integrated, since they are pure gauge, to give [12]-[14]

$$
A_{y}=D^{-1} D_{y}, A_{z}=D^{-1} D_{z}, A_{\bar{y}}=\bar{D}^{-1} \bar{D}_{\bar{y}}, A_{\bar{z}}=\bar{D}^{-1} \bar{D}_{\bar{z}},
$$


where $D$ and $\bar{D}$ are arbitrary $2 \times 2$ complex matrix functions of $y, \bar{y}, z$ and $\bar{z}$ , and with determinant $=1$ (for $\mathrm{SU}(2)$ gauge group) and $D_{y}=\partial_{y} D$, etc. For real gauge fields $A_{\mu} \doteq-A_{\mu}^{+}$(the symbol $\doteq$ is used for equations valid only for real values of $x_{1}, x_{2}, x_{3}$ and $x_{4}$ ), we require

$$
\bar{D} \doteq\left(D^{+}\right)^{-1} .
$$

Gauge transformations are the transformations

$$
D \rightarrow D U, \bar{D} \rightarrow \bar{D} U, U^{+} U \doteq I,
$$

where $U$ is a $2 \times 2$ matrix function of $y, \bar{y}, z, \bar{z}$ with determined $=1$. Under transformation (5), equation (4) remains unchanged. We now define the hermitian matrix $\jmath[15]-[17]$ as

$$
\jmath \equiv D \bar{D}^{-1} \doteq D D^{+},
$$

$\jmath$ has the very important property of being invariant under the gauge transformation (5). The only non vanishing field strengths in terms of $\jmath$ becomes

$$
F_{u \bar{v}}=-\bar{D}^{-1}\left(\jmath^{-1} \jmath_{u}\right)_{\bar{v}} \bar{D},
$$

$(u, v=y, z)$ and the remaining self-duality equation (2) takes the form

$$
\left(\jmath^{-1} \jmath_{y}\right)_{\bar{y}}+\left(\jmath^{-1} \jmath_{z}\right)_{\bar{z}}=0 .
$$

The action density in terms of $\jmath[18]$ is

$$
\begin{aligned}
\phi(\jmath) & =-\frac{1}{2} \operatorname{Tr} F_{\mu \nu} F_{\mu \nu} \\
& =-2 \operatorname{Tr}\left(F_{y \bar{y}} F_{z \bar{z}}+F_{y \bar{z}} F_{\bar{y} z}\right),
\end{aligned}
$$

where

$$
F_{\mu \nu}=-\left[D_{\mu}, D_{\nu}\right]=\partial_{\mu} A_{\nu}-\partial_{\nu} A_{\mu}-\left[A_{\mu}, A_{\nu}\right],
$$

where $F_{\mu \nu}$ are the gauge field strengths. Our construction begins by explicit parametrization of the matrix $\jmath$

$$
\jmath=\left(\begin{array}{cc}
\frac{1}{\phi} & \rho \\
\bar{\rho} & \phi(1+\rho \bar{\rho})
\end{array}\right),
$$

and for real gauge fields $A_{\mu} \doteq-A_{\mu}^{+}$,we require $\phi \doteq$ real,$\rho \doteq \rho^{*}\left(\rho^{*} \equiv\right.$ complex conjugate of $\rho$ ). The self-duality equations (8) take the form

$$
\frac{1}{2}(1+\rho \bar{\rho}) \partial_{\mu} \partial_{\mu} \ln \phi+\frac{1}{2} \rho \partial_{\mu} \partial_{\mu} \bar{\rho}+\frac{\bar{\rho}}{\phi}\left(\phi_{y} \rho_{\bar{y}}+\phi_{z} \rho_{\bar{z}}\right)
$$




$$
\begin{aligned}
& +\frac{\rho}{\phi}\left(\phi_{y} \bar{\rho}_{\bar{y}}+\phi_{z} \bar{\rho}_{\bar{z}}\right)+\left(\rho_{\bar{y}} \bar{\rho}_{y}+\rho_{\bar{z}} \bar{\rho}_{z}\right)=0, \\
& \frac{\phi}{2} \partial_{\mu} \partial_{\mu} \bar{\rho}+\frac{\bar{\rho}}{2} \partial_{\mu} \partial_{\mu} \phi-\frac{2 \bar{\rho}}{\phi}\left(\phi_{y} \phi_{\bar{y}}+\phi_{z} \phi_{\bar{z}}\right) \\
& +\left(\phi_{y} \bar{\rho}_{\bar{y}}+\phi_{z} \bar{\rho}_{\bar{z}}-\phi_{\bar{y}} \rho_{\bar{y}}-\phi_{\bar{z}} \rho_{\bar{z}}\right), \\
& \frac{\phi}{2} \partial_{\mu} \partial_{\mu} \rho+\frac{\rho}{2} \partial_{\mu} \partial_{\mu} \phi-\frac{2 \rho}{\phi}\left(\phi_{y} \phi_{\bar{y}}+\phi_{z} \phi_{\bar{z}}\right) \\
& +\left(\phi_{\bar{y}} \rho_{y}+\phi_{\bar{z}} \rho_{z}-\phi_{y} \rho_{\bar{y}}-\phi_{z} \rho_{\bar{z}}\right),
\end{aligned}
$$

where $\partial_{\mu} \partial_{\mu}=2\left(\partial_{y} \partial_{\bar{y}}+\partial_{z} \partial_{\bar{z}}\right)$. The positive definite Hermitian matrix $\jmath=$ $D D^{+}$can be factored into a product upper and lower (or vice versa) triangular matrices as follows

$$
\begin{gathered}
\jmath=R R^{+}=R^{I} R^{I+}, R=\left(\begin{array}{cc}
\frac{1}{\sqrt{\phi}} & 0 \\
\bar{\rho} \sqrt{\phi} & \sqrt{\phi}
\end{array}\right), R^{I}=\left(\begin{array}{cc}
\sqrt{\phi^{I}} & \bar{\rho}^{I} \sqrt{\phi^{I}} \\
0 & \frac{1}{\sqrt{\phi^{I}}}
\end{array}\right), \\
\phi \doteq \text { real }, \quad \bar{\rho} \doteq \rho^{*}, \quad \bar{\rho}^{I} \doteq \rho^{I *} .
\end{gathered}
$$

It is evident from (15) that one can choose a gauge so that $D=R$ or $D=R^{I}$ and it is easy to check that in both gauges the self-duality equations (12)-(14) (in the case of $D=R^{I}$ all the $\phi, \rho, \bar{\rho}$ are replaced by $\phi^{I}, \rho^{I}, \bar{\rho}^{I}$ ).

From equation (15) we see that $R^{-1} R^{I}$ is a unitary matrix so that we can always make a gauge transformation from the gauge $R$ to the $R^{I}$ gauge.

Theorem 1. If $(\phi, \rho, \bar{\rho})$ satisfy equations (12)- (14) then so do $\left(\phi^{I}, \rho^{I}, \bar{\rho}^{I}\right)$ defined by (see [19])

$$
\phi^{I}=\frac{1}{\phi}(1+\rho \bar{\rho}), \quad \rho^{I}=\bar{\rho}, \quad \bar{\rho}^{I}=\rho .
$$

Proof. By equating equation (15), we obtain the following equations

$$
\frac{1}{\phi}=\phi^{I}\left(1+\rho^{I} \bar{\rho}^{I}\right), \quad \rho=\bar{\rho}^{I}, \quad \bar{\rho}=\rho^{I}, \quad \phi\left(1+\rho^{I} \bar{\rho}^{I}\right)=\frac{1}{\phi^{I}} .
$$

We solve the system of equation (18) we obtain the relations in equation (17). 


\section{Exact Solution Class of the Classical SU(2) Yang-Mills Field Equations}

To obtain an exact solution class of the classical SU(2) Yang-Mills field equations in four-dimensional Euclidean space, consider the system.

$$
\begin{aligned}
& \frac{1}{2}(1+\rho \bar{\rho}) \partial_{\mu} \partial_{\mu} \ln \phi+\frac{1}{2} \rho \partial_{\mu} \partial_{\mu} \bar{\rho}+\frac{\bar{\rho}}{\phi}\left(\phi_{y} \rho_{\bar{y}}+\phi_{z} \rho_{\bar{z}}\right) \\
& +\frac{\rho}{\phi}\left(\phi_{y} \bar{\rho}_{\bar{y}}+\phi_{z} \bar{\rho}_{\bar{z}}\right)+\left(\rho_{\bar{y}} \bar{\rho}_{y}+\rho_{\bar{z}} \bar{\rho}_{z}\right)=0, \\
& \quad \frac{\phi}{2} \partial_{\mu} \partial_{\mu} \rho+\frac{\rho}{2} \partial_{\mu} \partial_{\mu} \phi-\frac{2 \rho}{\phi}\left(\phi_{y} \phi_{\bar{y}}+\phi_{z} \phi_{\bar{z}}\right) \\
& +\left(\phi_{\bar{y}} \rho_{y}+\phi_{\bar{z}} \rho_{z}-\phi_{y} \rho_{\bar{y}}-\phi_{z} \rho_{\bar{z}}\right) .
\end{aligned}
$$

Let us make the ansatz [20]

$$
\phi=\phi(g), \quad \rho=e^{i a} \sigma(g) .
$$

Where $g=g\left(x_{1}, x_{2}, x_{3}, x_{4}\right)$ is a real function of $x_{\mu}, \mu=1,2,3,4, \phi$ and $\sigma$ are real functions of $g$ and $a$ is a real constant. Then equations (19),(20) give the relations

$$
\begin{gathered}
\left(g_{y \bar{y}}+g_{z \bar{z}}\right)\left(\left(1+\sigma^{2}\right) \phi^{2}\right)^{\prime}-2\left(g_{y} g_{\bar{y}}+g_{z} g_{\bar{z}}\right) \phi^{2}\left[\left(1+\sigma^{2}\right) \frac{\phi^{\prime}}{\phi}+\sigma \sigma^{\prime}\right]^{\prime}=0 \\
\left(g_{y \bar{y}}+g_{z \bar{z}}\right)(\phi \sigma)^{\prime}+\left(g_{y} g_{\bar{y}}+g_{z} g_{\bar{z}}\right) \phi^{2}\left[\frac{(\phi \sigma)^{\prime}}{\phi^{2}}\right]^{\prime}=0
\end{gathered}
$$

Where the prime means differentiation with respect to $g$. The above relations imply that the determinant of the coefficients of $\left(g_{y \bar{y}}+g_{z \bar{z}}\right)$ and $\left(g_{y} g_{\bar{y}}+g_{z} g_{\bar{z}}\right)$ is zero i.e.

$$
\left(\left(1+\sigma^{2}\right) \phi^{2}\right)^{\prime}\left[\frac{(\phi \sigma)^{\prime}}{\phi^{2}}\right]^{\prime}+2\left[\left(1+\sigma^{2}\right) \frac{\phi^{\prime}}{\phi}+\sigma \sigma^{\prime}\right]^{\prime}(\phi \sigma)^{\prime}=0 .
$$

We shall determine $\phi$ and $\sigma$ from the above equation $(24)$, let $(\phi \sigma)=c$, where $\mathrm{c}$ is a constant, then $(\phi \sigma)^{\prime}=0$,

$$
\left(\left(1+\sigma^{2}\right) \phi^{2}\right)^{\prime}\left[\frac{(\phi \sigma)^{\prime}}{\phi^{2}}\right]^{\prime}=0,\left[\left(1+\sigma^{2}\right) \frac{\phi^{\prime}}{\phi}+\sigma \sigma^{\prime}\right]^{\prime}(\phi \sigma)^{\prime}=0 .
$$

We suppose

$$
\phi=\sqrt{c} e^{-g}, \sigma=\sqrt{c} e^{g} \text {, then } \rho=\sqrt{c} e^{g+i a} .
$$


Applying theorem (1) to $\phi$ and $\rho$ of equation (26), then we get

$$
\phi^{I}=\frac{e^{g}}{\sqrt{c}\left(1+c e^{2 g}\right)}, \quad \rho^{I}=\sqrt{c} e^{g-i a}, \quad \bar{\rho}^{I}=\sqrt{c} e^{g+i a} .
$$

Equations (26) and (27) is a new class of solutions of Yang-Mills equations for self-dual SU(2)gauge fields.

\section{Exact Solutions for Self-Dual $S U(2)$ Gauge Fields on Euclidean Space, when $\rho$ is a Complex Analytic Function, see [21]}

We reduce the equations for self-dual $S U(2)$ gauge fields on Euclidean space to the following equations

$$
\begin{aligned}
& \frac{1}{2}(1+\rho \bar{\rho}) \partial_{\mu} \partial_{\mu} \ln \phi+\frac{1}{2} \rho \partial_{\mu} \partial_{\mu} \bar{\rho}+\frac{\bar{\rho}}{\phi}\left(\phi_{y} \rho_{\bar{y}}+\phi_{z} \rho_{\bar{z}}\right) \\
& +\frac{\rho}{\phi}\left(\phi_{y} \bar{\rho}_{\bar{y}}+\phi_{z} \bar{\rho}_{\bar{z}}\right)+\left(\rho_{\bar{y}} \bar{\rho}_{y}+\rho_{\bar{z}} \bar{\rho}_{z}\right)=0 \\
& \frac{\phi}{2} \partial_{\mu} \partial_{\mu} \rho+\frac{\rho}{2} \partial_{\mu} \partial_{\mu} \phi-\frac{2 \rho}{\phi}\left(\phi_{y} \phi_{\bar{y}}+\phi_{z} \phi_{\bar{z}}\right) \\
& +\left(\phi_{\bar{y}} \rho_{y}+\phi_{\bar{z}} \rho_{z}-\phi_{y} \rho_{\bar{y}}-\phi_{z} \rho_{\bar{z}}\right) .
\end{aligned}
$$

When $\rho$ is a complex analytic function of $y$ and $z$, then we have

$$
\rho_{\bar{y}}=\bar{\rho}_{z}=0 \quad, \quad \rho_{y} \rho_{\bar{y}}+\rho_{z} \rho_{\bar{z}}=0 .
$$

Then, the self-dual Yang-Mills equations(28),(29)takes the form

$$
\begin{gathered}
\phi\left(\phi_{y \bar{y}}+\phi_{z \bar{z}}\right)-\left(\phi_{y} \phi_{\bar{y}}+\phi_{z} \phi_{\bar{z}}\right)=0, \\
\rho\left(\phi_{y \bar{y}}+\phi_{z \bar{z}}\right)-2 \frac{\rho}{\phi}\left(\phi_{y} \phi_{\bar{y}}+\phi_{z} \phi_{\bar{z}}\right)+\left(\rho_{y} \phi_{\bar{y}}+\rho_{z} \phi_{\bar{z}}\right)=0 .
\end{gathered}
$$

We consider now two cases:

(a) Let $\rho=\rho(\phi)$, then we find

$$
\rho_{y}=\rho^{\prime} \phi_{y} \quad, \quad \rho_{z}=\rho^{\prime} \phi_{z} .
$$

By using equation(33), then the two equations(31)and(32)become

$$
\phi\left(\phi_{y \bar{y}}+\phi_{z \bar{z}}\right)-\left(\phi_{y} \phi_{\bar{y}}+\phi_{z} \phi_{\bar{z}}\right)=0,
$$




$$
\rho\left(\phi_{y \bar{y}}+\phi_{z \bar{z}}\right)-\left(\rho^{\prime}-2 \frac{\rho}{\phi}\right)\left(\phi_{y} \phi_{\bar{y}}+\phi_{z} \phi_{\bar{z}}\right)=0 .
$$

If we do not consider the cases $\left(\phi_{y \bar{y}}+\phi_{z \bar{z}}\right)=0$ and $\left(\phi_{y} \phi_{\bar{y}}+\phi_{z} \phi_{\bar{z}}\right)=0$, then we have

$$
\phi \rho^{\prime}-\rho=0
$$

by integration we obtain

$$
\rho=c \phi
$$

where $\mathrm{c}$ is a complex constant both equations (34) and (35) reduce to the same equation. A solution is given by

$$
\phi_{y}=\phi_{z} \quad, \phi_{\bar{y}}=-\phi_{\bar{z}} .
$$

The solution class is given by

$$
\phi=F(y+z, \bar{y}-\bar{z}),
$$

where $F$ is an arbitrary function, equations (37) and (39) gives a new class of solutions of Yang-Mills equations for self-dual $S U(2)$ gauge fields. Applying theorem (1) to $\phi$ and $\rho$ of equations (37) and (39), then we get

$$
\phi^{I}=\frac{1}{F\left(1+c \bar{c} F^{2}\right)}, \quad \rho^{I}=\bar{c} F, \quad \bar{\rho}^{I}=c F .
$$

(b) Let us make the ansatz [20]

$$
\phi=\phi(g), \quad \rho=e^{i a} \sigma(g) .
$$

Where $g=g\left(x_{1}, x_{2}, x_{3}, x_{4}\right)$ is a real function of $x_{\mu}, \mu=1,2,3,4, \phi$ and $\sigma$ are real functions of $g$ and $a$ is a real constant. Then equations (31),(32) give the relations

$$
\begin{array}{r}
\phi \phi^{\prime}\left(g_{y \bar{y}}+g_{z \bar{z}}\right)+\left(g_{y} g_{\bar{y}}+g_{z} g_{\bar{z}}\right)\left[\phi \phi^{\prime \prime}-\left(\phi^{\prime}\right)^{2}\right]=0 \\
\left.\sigma \phi^{\prime}\left(g_{y \bar{y}}+g_{z \bar{z}}\right)+\left(g_{y} g_{\bar{y}}+g_{z} g_{\bar{z}}\right)\left[\sigma \phi^{\prime \prime}-2 \frac{\sigma\left(\phi^{\prime}\right)^{2}}{\phi}+\phi^{\prime} \sigma^{\prime}\right)\right]=0 .
\end{array}
$$

Where the prime means differentiation with respect to $g$. The above relations imply that the determinant of the coefficients of $\left(g_{y \bar{y}}+g_{z \bar{z}}\right)$ and $\left(g_{y} g_{\bar{y}}+g_{z} g_{\bar{z}}\right)$; is zero i.e.

$$
\frac{\sigma^{\prime}}{\sigma}=\frac{\phi^{\prime}}{\phi}
$$


by integration (44) we obtain

$$
\sigma(g)=c \phi(g), \quad \rho=c e^{i a} \phi(g) .
$$

Applying theorem (1) to $\phi$ and $\rho$ of equation (45), then we get

$$
\phi^{I}=\frac{1}{\phi(g)\left(1+c^{2} \phi^{2}(g)\right)}, \quad \rho^{I}=c e^{-i a} \phi(g), \quad \bar{\rho}^{I}=c e^{i a} \phi(g) .
$$

Equations (45) and (46) is a new class of solutions of Yang-Mills equations for self-dual $S U(2)$ gauge fields.

\section{Conclusions}

A new class of solutions of Yang-Mills equations for self-dual $S U(2)$ gauge fields are investigated. In this paper we found a new representation for self-duality equations. In addition exact solution class of the classical $S U(2)$ Yang-Mills field equations in four-dimensional Euclidean space and two exact solution classes for $S U(2)$ Yang-Mills equations when $\rho$ is a complex analytic function are also obtained.

\section{References}

[1] A.H. Khater, D.K. Callebaut, A.A. Abdalla and S.M. Sayed, Exact solutions for self-dual Yang-Mills Equations, Chaos Solitons and Fractals, Vol.10, (1999), pp. 1309-1320.

[2] A.H. Khater, D.K. Callebaut, A.A. Abdalla, A.R. Shehata and S.M. Sayed, Bäcklund transformations and exact solutions for self-dual SU(3) YangMills equations, IL Nuovo Cimento B, Vol.114, No1,(1999), pp.1-10.

[3] A.H. Khater and S.M. Sayed, Exact Solutions for Self-Dual SU(2) and SU(3)Yang-Mills Fields, International J. of Theoretical Physics, Vol.41, (2002), pp. 409-419.

[4] M.J. Ablowitz, S. Chakravarty and R. Halburd, Integrable systems and reductions of the self-dual Yang-Mills equations, J. Math. Phys., Vol.44, (2003), pp. 3147-3173.

[5] R. Rajaraman, Solitons and Instantons: An Introduction to Solitons and Instantons in Quantum Field Theory , (North-Holland, Amsterdam), (1989). 
[6] L. J. Mason and E. T. Newman, A connection betweenthe Einstein and Yang-Mills equations, Commun. Math. Phys, Vol.121, (1989), pp. 659668.

[7] L. Witten, Static axially symmetric solutions of self dual SU(2) gauge fields in Euclidean four-dimensional space, Phys. Rev.D, Vol. 19, (1979), pp. 718-720.

[8] S. K. Donaldson, An application of gauge theory to the topology of 4 manifolds, J. Diff. Geom., Vol. 18, (1983), pp. 279-315.

[9] E. Corrigan, Self dual solutions to Euclidean Yang-Mills equations, Phys. Rep., Vol. 49, (1979), pp. 95-111.

[10] E. Corrigan, Static non-abelian forces and the permutation group, Phys. Letts.B, Vol. 82, (1979), pp. 407-410.

[11] C.N. Yang and R.L. Mills, Conservation of isotopic spin and isotopic gauge invariance, Phys. Rev., Vol.96, (1954), pp. 191-195.

[12] L. L. Chau and I. Yamanaka, Canonical formulation of the self-dual YangMills system: Algebras and Hierarchies, phys. Rev. lett., Vol. 68, (1992), pp. $180 \%-1810$.

[13] L. L. Chau and I. Yamanaka, Quantization of the self-dual Yang- Mills system:Exchange Algebras and local Quantum group in four-dimensional Quantum field theories, phys. Rev. lett., Vol. 70, (1993), pp.1916-1919.

[14] M. L.Ge, L. Wang and Y. S. Wu, Canonical reduction of self-dual Yang-Mills theory to sine-Gordon and Liouville theories, phys.Lett.B, Vol.335,(1994), pp. 136-142.

[15] E. Corrigan, D. Fairlie, P. Goddard and R. G. Yates, The construction of self-dual solutions to SU(2) gauge theory,Commums. Math. Phys., Vol.58,(1978), pp. 223-240.

[16] E. Corrigan , P. Goddard and S. Templeton, Instanton Green functions and tensor products, Nucl. Phys.B, Vol.151,(1979), pp. 93-117.

[17] E. Corrigan, C. Devchand, D. Fairlie and J. Nuyts, First order equations for gauge theories in dimension greater than four, Nucl. Phys.B, Vol. 214, (1983), pp. 452-464. 
[18] E. Corrigan and B. Hasslacher, A functional equation for exponential loop integrals in gauge theories, Phys. Letts.B, Vol. 81 ,(1979) , pp. 181-184.

[19] M. K. Prasad,Instantons and Monopols in Yang-Mills gauge field theories, Physica D, Vol. 1, (1980), pp. 167-191.

[20] E. Kyriakopoulos,Solutions of the Yang - Mills field equations, Phys. Lett.B, Vol.95 ,(1980), pp. 409-412.

[21] A. H. Khater, A. R. Shehatah, D. K. Callebaut and S. M. Sayed, SelfDual Solutions for SU(2) and SU(3) Gauge Fields on Euclidean Space, International J. of Theoretical Physics, Vol. 43 (2004), pp. 151-159. 